\title{
Testing levelness error of long cylindrical guide rail by laser tracker
}

\author{
Yiliu Xu ${ }^{1,2}$, Yong Lu ${ }^{3, *}$, Tao Guo ${ }^{1,2}$, Weizhi Zheng ${ }^{1,2}$ \\ ${ }^{1}$ Beijing Institute of Spacecraft Environment Engineering, Beijing 100094, China \\ ${ }^{2}$ Beijing Engineering Research Center of the Intelligent Assembly Technology and Equipment for \\ Aerospace Product, Beijing 100094, China \\ ${ }^{3}$ School of Mechatronics Engineering, Harbin Institute of Technology, Harbin, 150001, China \\ *Luyhit@163.com
}

Key words: laser tracker, levelness, long cylindrical guide rail, detection

\begin{abstract}
Laser tracker has been widely applied in the field of long rails geometric tolerance measurement due to high precision, ease of use and other reasons. Round rail levelness measurement is used to be calculated by means of electronic level using the pitch method which is more cumbersome and error-prone. Based on the analysis of laser tracker leveling principle, we propose a new method using laser tracker measuring the level of the long cylindrical guide rail. With a project example, cylindrical guide rail located at altitude of $5 \mathrm{~m}$ and $16 \mathrm{~m}$ in length were proceeded for level measurement and the measured level of the long cylindrical guide rail is $0.03 \mathrm{~mm} / \mathrm{m}$. The method broadened the field using a laser tracker. By using the laser tracker measurement principle in long long cylindrical guide rails levelness, it can also be applied to the parallelism, squareness, inclination and other measurement.
\end{abstract}

\section{Introduction}

Circular guide levelness is usually read directly by using electronic level meter. The method has its limits for the levelness measurement of the super-long cylindrical guide rail, the super-long cylindrical guide rail needs to be measured in sections, the graphical method is used to calculate the levelness error, so there is a large error in the measurement and calculation. ${ }^{[1]}$ To solve the limits of traditionally measuring cylindrical guide rail levelness, this paper puts forward the method of using laser tracker to measure the super-long cylindrical guide rails levelness. The horizontal datum is established through the horizontal inclination compensation function of the laser tracker, the horizontal work coordinate system is established on the basis of the horizontal datum, the special self-centering target holder is designed to fix the target and measure the space coordinates of super-long cylindrical guide rails, and the least-square fitting method is used to obtain the fitting straight line of the measuring point, to calculate the levelness of the super-long guide rail. Compared with the measurement method of the original guide levelness, the method improves the measurement accuracy, reduces the calculation error and the measuring time.

\section{Measuring principle}

The measuring principle of super-long cylindrical guide rail levelness by laser tracker as shown in Fig.1. It consists of the laser tracking head, tripod, super-long cylindrical guide rail, self-centering target holder and spherically mounted retro reflectors(SMR). 


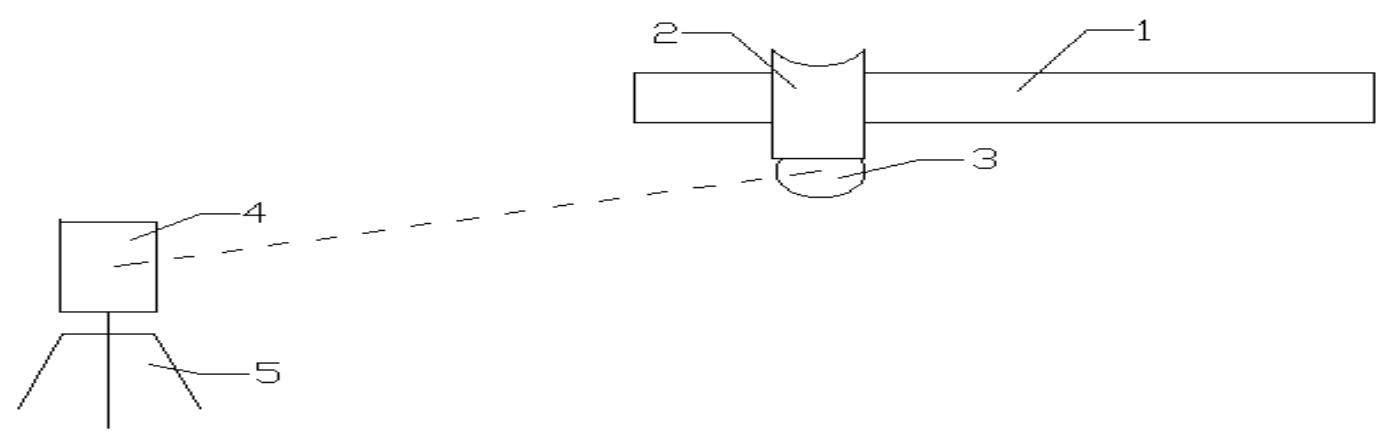

$\begin{array}{llll}\text { 1. super-long cylindrical guide rail } & \text { 2. self-centering target holder } & 3 . \text { SMR } & \text { 4.laser tracker } \quad \text { 5. tripod }\end{array}$

Figure 1 Schematic drawing of Testing levelness error of super-long cylindrical guide rail

Laser tracker is used to measure the spatial position, with the characteristics of high measurement accuracy, fast response speed, large measurement range, etc. ${ }^{[2]}$ The core component of laser tracker is laser tracking head, which contains the distance measurement, angle measurement, tracking control and other functions. Its distance measuring part of API T3 model laser tracker includes interferometry measurement(IFM) and absolute distance measurement(ADM) respectively, a He-Ne laser and an infrared laser are emitted with the help of the laser tracking head for measurement, the laser beam returns to the tracking head through SMR reflection, and the spatial position of SMR is parsed through the angle measurement. Dual axis lateral effect photodetector receives the changes of laser beam position when the SMR moves, and the motor driven pitching angle (EL) axis and azimuth (AZ) axis realize the tracking of SMR.

To make it convenient for the levelness measurement tracker of the super-long cylindrical guide rail to adopt the absolute distance measurement(ADM) mode, the measurement accuracy of absolute distance measurement (ADM) mode of API T3 model laser tracker is $15 \mu \mathrm{m}+\mathrm{L}^{*} 1.5 \mu \mathrm{m}$ ( $\mathrm{L}$ is greater than $10 \mathrm{~m}$ ), and the measurement accuracy is $15 \mu \mathrm{m}$ when $\mathrm{L}$ is less than $10 \mathrm{~m}$, $\mathrm{L}$ is the distance between laser tracking head and SMR. The side-tipping sensor is installed in the tracking head of laser tracker, which can measure the relative angle between laser tracking head and the horizontal plane, and the angle value is read through the measurement software Spatial Analyzer. When the measurement accuracy of the side-tipping sensor is $a$, the levelness error of the super-long guide rail is $1000 * \tan a(\mathrm{~mm} / \mathrm{m})$; the measurement accuracy of API T3 model laser tracker is 3", the levelness error of the measured super-long cylindrical guide rail is $0.015 \mathrm{~mm} / \mathrm{m}$.

The horizontal work coordinate is needed before testing levelness. The process of the establishment of the horizontal work coordinate of laser tracker is described below.

(1) Calculate reference value $a$

Laser tracking head is rotated to any location, where the horizontal inclination value $v$ is read in the Spatial Analyzer measuring software. When the laser tracking head is rotated $180^{\circ}$, now the horizontal inclination value $w$ is read. The value of the side-tipping sensor of the horizontal situation of the laser tracker level is, namely basic value $a=(v+w) / 2$.

(2) Rotate $\mathrm{x}$ axis

Aim the laser tracking head at any leg in the laser tracker shelf bracket stand, adjust the adjusting knob of the rest of two legs, until the horizontal inclination reading is near $a$.

(3) Rotate y axis

Rotate the laser tracking head $90^{\circ}$, adjust the leg not adjusted in the step (2), until the horizontal inclination reading is near $a$.

(4) Determination of horizontal inclination 
Rotate the laser tracking head $360^{\circ}$, determine whether the horizontal angle change amplitude meets the requirements of horizontal inclination compensation.

(5) Angular-error compensation

The horizontal inclination values of two directions differing $90^{\circ}$ are measured, and the measured space coordinate value of the laser tracker in the actual coordinate system is transformed to the horizontal coordinate system based on the coordinate transformation theory. The function is integrated in Spatial Analyzer, so it can be directly called to complete angular-error compensation and generate the horizontal coordinate system ${ }^{[3]}$.

(6) Establish the horizontal work coordinate system

$\mathrm{z}$ axis of horizontal coordinates is taken as the $\mathrm{z}$ axis of the horizontal coordinate system, and the super-long circular guide axial direction as $\mathrm{x}$ axis to establish the horizontal work coordinate system.

\section{Detection experiment}

Combined with engineering practice, levelness measurement is conducted on the super-long circular guide with the length of $16 \mathrm{~m}$ in the sky with the height of $5 \mathrm{~m}$, the target of the laser tracker is fixed on a special self-centering target holder, as shown in Fig.2. Special target holder contacts the circular guide through two pulleys, which can rotate around the circular guide optionally with low friction. Since it has self-centering and larger quality, this ensures the position of target lens center doesn't develop offset when it moves slowly along the guide rail, so as to reduce the measurement error.

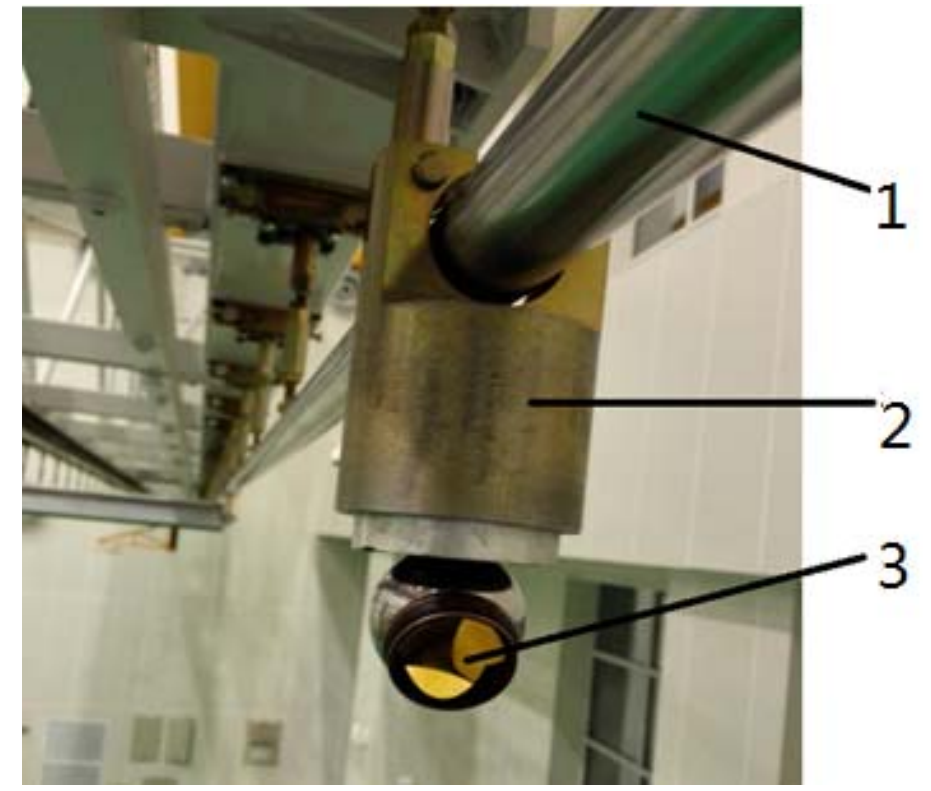

$\begin{array}{lll}\text { 1. super-long cylindrical guide rail } & \text { 2. special self-centering target holder } & 3 \text {. SMR }\end{array}$ Figure 2 Pecial self-centering target holder

The space coordinates of 38 points are collected evenly on the super-long cylindrical guide of $16 \mathrm{~m}$. The least square method is used to simulate to the sampling point and is projected onto the XOZ plane, as shown in Fig.3. The levelness of super-long circular guide is $0.03 \mathrm{~mm} / \mathrm{m}$, and normal distribution curve of test points is as shown in Fig.4. 


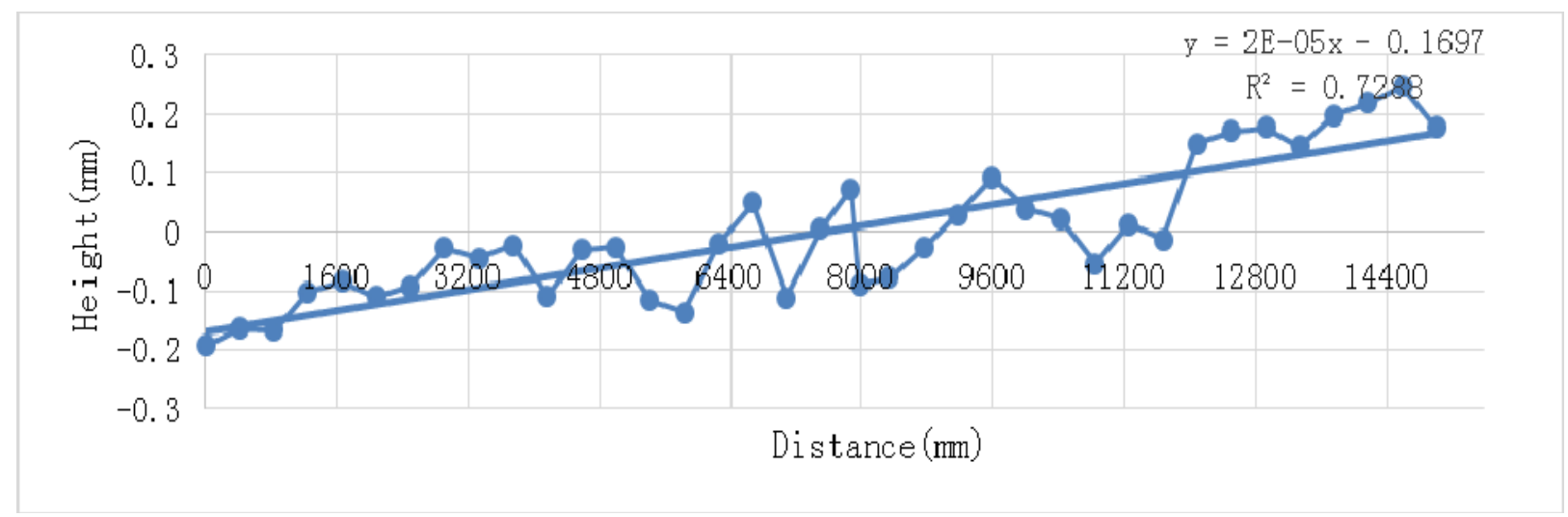

Figure 3 Best-line fit of projected points

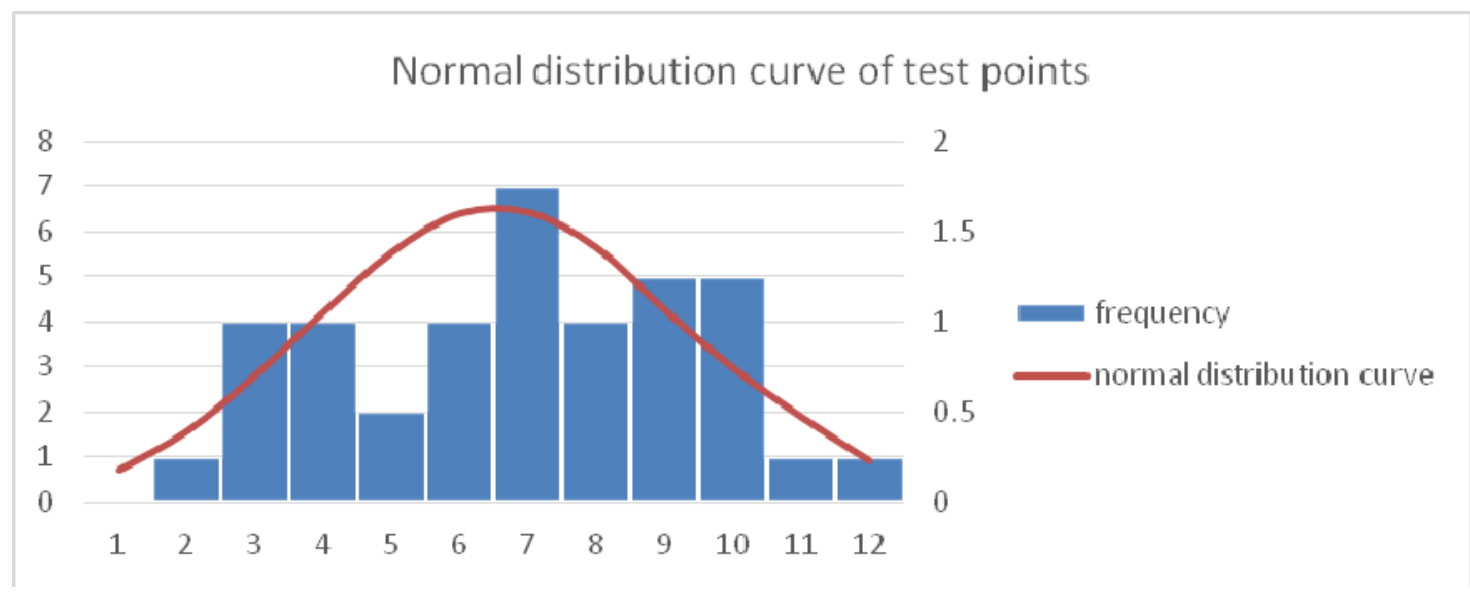

Figure 4 Normal distribution curve of test points

\section{Summary}

This paper puts forward the method to establish the horizontal coordinate system of laser tracker, designs the special self-centering target holder suitable for circular guide measurement and completes the levelness measurement of the super-long cylindrical guide rail with the length of $16 \mathrm{~m}$ based on this. The measured levelness of the super-long cylindrical guide rail is $0.03 \mathrm{~mm} / \mathrm{m}$. The laser tracker is used to measure the principle of super-long cylindrical guide rail levelness, and it also can be applied to the measurement of guide rail parallelism, perpendicularity and inclination.

\section{References}

[1] KNAPP W, BRINGMANN B. Straightness Measurement for Long Movements [J]. Nanotechnology and Precision Engineering,2005,04:249-256.

[2]Wang xiao-kun.Testing linearity error of long guideway by laser tracker[J].Journal of Applied Optics, 2013,04:686-689.(in Chinese)

[3] LU Rong-sheng, LI Wan-hong, LAO Da-bao. Angular error compensation for laser tracker [J]. Optics and Precision Engineering,2014,09:2299-2305. (in Chinese) 